\title{
THE FUGLEDE COMMUTATIVITY THEOREM MODULO OPERATOR IDEALS
}

\author{
GARY WEISS
}

\begin{abstract}
Let $\boldsymbol{H}$ denote a separable, infinite-dimensional complex Hilbert space. A two-sided ideal $\boldsymbol{I}$ of operators on $\boldsymbol{H}$ possesses the generalized Fuglede property (GFP) if, for every normal operator $N$ and every $X \in L(H), N X-X N \in I$ implies $N^{*} X-X N^{*} \in I$. Fuglede's Theorem says that $I=\{0\}$ has the GFP. It is known that the class of compact operators and the class of Hilbert-Schmidt operators have the GFP.

We prove that the class of finite rank operators. and the Schatten $p$-classes for $0<p<1$ fail to have the GFP. The operator we use as an example in the case of the Schatten $p$-classes is multiplication by $z+w$ on $L^{2}$ of the torus.
\end{abstract}

Introduction. Let $\boldsymbol{H}$ denote a separable, infinite-dimensional complex Hilbert space. Let $L(H) \supset K(H) \supset C_{p} \supset F(H)(0<p<\infty)$ denote, respectively, the class of all bounded linear operators, the class of compact operators, the Schatten $p$-class, and the class of finite rank operators on $H$. All operators herein are assumed to be linear and bounded. Let $\|\cdot\|_{p}$ denote the $C_{p}$-norm.

Let $I$ be any two-sided ideal in $L(H)$ (every ideal herein is assumed to be two-sided). It is well known that if $I \neq\{0\}$ or $L(H)$, then $K(H) \supset I \supset F(H)$.

Definition. The ideal $I$ is said to possess the generalized Fuglede property (GFP) if, for every normal operator $N$ and every bounded operator $X$, we have $N X-X N$ $\in I$ implies $N^{*} X-X N^{*} \in I$ (i.e., $N X=X N$ modulo $I$ implies $N^{*} X=X N^{*}$ modulo $I$ ).

Fuglede's Theorem essentially states that $I=\{0\}$ has the GFP [1].

There is a connection between the GFP for $C_{1}$ (which is not known to hold true) and a possible generalization of the trace result [5, Question 3]. Namely, if $N$ is a normal operator and $X$ is a compact operator such that $N X-X N \in C_{1}$, must $\operatorname{trace}(N X-X N)=0$ ?

If $C_{1}$ possessed the GFP, then the answer to this question would be yes. The proof is the same as the proof for the well-known fact that if $S$ is a selfadjoint operator and $X$ is a compact operator then $S X-X S \in C_{1}$ implies $\operatorname{trace}(S X-X S)=0$ (cf. [8, p. 279, Lemma 1.3]). Say $T=N X-X N \in C_{1}$. If $C_{1}$ possessed the GFP, then $S=N^{*} X-X N^{*} \in C_{1}$. Hence $-S^{*}=N X^{*}-X^{*} N \in$ $C_{1}$ and $T-S^{*}=N\left(X+X^{*}\right)-\left(X+X^{*}\right) N$ and $T+S^{*}=N\left(X-X^{*}\right)-$ $\left(X-X^{*}\right) N$. But $X+X^{*}$ and $X-X^{*}$ are scalar multiples of compact, selfadjoint

Received by the editors February 13, 1980 and, in revised form, May 19, 1980 and August 27, 1980. 1980 Mathematics Subject Classification. Primary 47A30, 47B05, 47B10, 47B15, 47B47; Secondary 47A55, 47A05, 47D25.

Key words and phrases. Commutator, normal operator, normal derivation, operator ideal, Fuglede property, Schatten $p$-norms. 
operators. By the spectral theorem they are diagonalizable. By a computation the above commutators must have trace 0 . Therefore

$$
\operatorname{trace}\left(T-S^{*}\right)=\operatorname{trace}\left(T+S^{*}\right)=0 .
$$

Hence trace $T=0$.

With these remarks, we pose the following questions:

1. Does $C_{1}$ possess the GFP?

2. If $N$ is normal and $X$ is compact, does $N X-X N \in C_{1}$ imply $\operatorname{trace}(N X-X N)=0$ ?

3. Are there any ideals in $L(H)$ other than $\{0\}, C_{2}$ and $K(H)$ that possess the GFP?

Some positive results. Let $|T|$ denote $\sqrt{T^{*} T}$.

The first interesting observation is that if $I$ is any ideal and $N$ is normal and $X \in L(H)$, then $|N X|=\left|N^{*} X\right|$. This is a trivial computation. In fact, if $N$ is hyponormal, then $|N X| \geqslant\left|N^{*} X\right|$. From this we get $N$ hyponormal implies $N X \in I$ $\Rightarrow N^{*} X \in I$. Furthermore if $N$ is normal and $I$ is a normed ideal where $\|T\|_{I}=$ $\||T|\|_{I}$ for all $T \in I$, then $\|N X\|_{I}=\left\|N^{*} X\right\|_{I}$. The same holds for $X N$ and $X N^{*}$.

The compacts $K(H)$ have the GFP. This is clear by applying Fuglede's Theorem to the Calkin algebra.

The Class $C_{2}$ has the GFP [6, Theorem 1]. (For another proof use Voiculescu's Theorem [3].)

The Putnam generalization of Fuglede's Theorem [2] applies to the GFP. That is any ideal $I$ has the GFP if and only if for every two normal operators $N_{1}, N_{2}$ and $X \in L(H), N_{1} X-X N_{2} \in I$ implies $N_{1} X-X N_{2} \in I$. This is proved in an analogous way to the well-known proof of Putnam's generalization [2] using a $2 \times 2$ matrix trick due to S. K. Berberian [7].

\section{The counterexamples.}

THEOREM 1. The class of finite rank operators $F(H)$ does not possess the GFP.

Proof. By the previous remark, it suffices to produce two trace class diagonal operators $D_{1}, D_{2}$ and a Hilbert-Schmidt operator $X$ such that the rank of $D_{1} X-$ $X D_{2}$ is 1 , but the rank of $D_{1}^{*} X-X D_{2}^{*}$ is infinite.

Let $D_{1}$ denote the diagonal matrix with diagonal entries $1 / 2,1 / 4,1 / 8, \ldots$ Clearly, $D_{1} \in C_{1}$. Formally let $D_{2}$ denote the diagonal matrix with diagonal entries $z_{1}, z_{2}, \ldots$ and $X_{1}=\left(x_{i j}\right)$ where $x_{i j}=4^{-(i+j)}\left(2^{-i}-z_{j}\right)^{-1}$. We shall choose the sequence $\left(z_{n}\right)$ inductively to satisfy several conditions. First of all, we want to be sure that $\left|2^{-i}-z_{j}\right|$ is large enough to insure that $X_{1} \in C_{2}$, that is, $x_{i j}$ is square summable. Secondly, we need to be sure that $\left|z_{n}\right|$ is small enough to insure that $D_{2} \in C_{1}$.

In the first place, if we can choose $\left(z_{n}\right)$ to be in the closed, left half-plane, then $\left|2^{-i}-z_{j}\right| \geqslant 2^{-i}$, and so $\left|x_{i j}\right| \leqslant 4^{-(i+j)} 2^{i}=2^{-i} 4^{-j}$ which is square summable.

In the second place, we can insure that $D_{2} \in C_{1}$ if we can choose $\left(z_{n}\right)$ such that $\left|z_{n}\right| \leqslant 2^{-n}$. 
In regard to the rank requirements on $D_{1} X_{1}-X_{1} D_{2}$ and $D_{1}^{*} X_{1}-X_{1} D_{2}^{*}$, note that

$$
\left(D_{1} X_{1}-X_{1} D_{2}\right)(i, j)=\left(2^{-i}-z_{j}\right) 4^{-(i+j)}\left(2^{-i}-z_{j}\right)^{-1}=4^{-(i+j)},
$$

and so the range of $D_{1} X_{1}-X_{1} D_{2}$ is the 1-dimensional subspace spanned by the vector $(1,1 / 4,1 / 16, \ldots)$. Therefore, since

$$
\left(D_{1}^{*} X_{1}-X_{1} D_{2}^{*}\right)(i, j)=4^{-(i+j)}\left(\left(2^{-i}-\bar{z}_{j}\right) /\left(2^{-i}-z_{j}\right)\right),
$$

it is clear that $D_{1}^{*} X_{1}-X_{1} D_{2}^{*}$ has an infinite-dimensional range provided that we choose the sequence $\left(z_{n}\right)$ with one additional property. That is, for each positive integer $N$, the $N$ vectors given by $4^{-(i+j)}\left(\left(2^{-i}-\bar{z}_{j}\right) /\left(2^{-i}-z_{j}\right)\right)_{i=1}^{\infty}$ for $1 \leqslant j \leqslant N$ form a linearly independent set. Clearly, for this to hold, it is sufficient that these $N$ vectors be linearly independent in the first $N$ coordinates. Equivalently, it is sufficient to show that the determinant of the corresponding $N \times N$ matrix be nonzero; that is, it suffices to prove that there exists a sequence $\left(z_{n}\right)$ in the closed left half-plane for which $\left|z_{n}\right|<2^{-n}$ for every $n$ and for which

$$
\operatorname{det}\left(4^{-(i+j)}\left(\left(2^{-i}-\bar{z}_{j}\right) /\left(2^{-i}-z_{j}\right)\right)\right)_{i, j=1}^{N} \neq 0
$$

for every $N$. We prove this by induction.

Let $z_{1}=0$. Then the case $N=1$ is trivial. (Observe that $N=2$ means $\operatorname{dim} H=$ 4; it is curious to note and easy to prove that if $\operatorname{dim} H=3$ then $\operatorname{rank}(D X-X D)$ $=\operatorname{rank}\left(D^{*} X-X D^{*}\right)$.)

Assume $\left\langle z_{n}\right\rangle_{n=1}^{N}$ has been chosen to satisfy the induction hypothesis. Let $z_{N+1}$ denote the free complex variable which ranges over the intersection of the open, left half-plane and the open disc $\left[|z|<2^{-(N+1)}\right]$. Then

$$
\begin{aligned}
f\left(z_{N+1}, \bar{z}_{N+1}\right) & =\operatorname{det}\left(4^{-(i+j)}\left(\left(2^{-i}-\bar{z}_{j}\right) /\left(2^{-i}-z_{j}\right)\right)\right)_{i, j=1}^{N+1} \\
& =\sum_{i=1}^{N+1}(-1)^{N+1-i} D_{i} 4^{-(i+N+1)}\left(\left(2^{-i}-\bar{z}_{N+1}\right) /\left(2^{-i}-z_{N+1}\right)\right),
\end{aligned}
$$

where $D_{i}$ is the subdeterminant of the $(i, N+1)$ entry which is

$$
4^{-(i+N+1)}\left(\left(2^{-i}-\bar{z}_{N+1}\right) /\left(2^{-i}-z_{N+1}\right)\right) \text {. }
$$

Clearly, by inspection and the induction hypothesis,

$$
D_{N+1}=\operatorname{det}\left(4^{-(i+j)}\left(\left(2^{-i}-\bar{z}_{j}\right) /\left(2^{-i}-z_{j}\right)\right)\right)_{i, j=1}^{N} \neq 0
$$

and we will use this fact presently. If we let $a_{i}=(-1)^{N+1-i} D_{i} 4^{-(i+N+1)}$ and $z=z_{N+1}$, then it suffices to show that there exists a $z$ contained in the intersection of the open, left half-plane and the open disc $\left[|z|<2^{-(N+1)}\right]$ such that

$$
f(z, \bar{z})=\sum_{i=1}^{N+1} a_{i}\left(\left(2^{-i}-\bar{z}\right) /\left(2^{-i}-z\right)\right) \neq 0 .
$$

To see that such a complex number $z$ exists, suppose to the contrary that $f(z, \bar{z})=0$ in this region. Taking the $\bar{z}$ derivative of both sides of this equation, we obtain $0=f_{\bar{z}}(z, \bar{z})=\sum_{i=1}^{N+1}-a_{i} /\left(2^{-i}-z\right)$, for every $z$ in this region. However, 
$a_{N+1}=D_{N+1} 4^{-2(N+1)} \neq 0$ and so $f_{\bar{z}}(z, \bar{z})=\sum_{i=1}^{N+1}-a_{i} /\left(2^{-i}-z\right)$ becomes unbounded in the open disc $\left[|z|<2^{-(N+1)}\right]$ near the point $z=2^{-(N+1)}$. Therefore, $f_{\bar{z}}(z, \bar{z})$ is not identically 0 in this open disc. But since $f_{\bar{z}}(z, \bar{z})=0$ throughout the intersection of the open, left half-plane and the open disc, we must have that $f_{\bar{z}}(z, \bar{z})$ is identically 0 in this open disc, which is a contradiction. Q.E.D.

We now present the result of the Schatten $p$-classes. Let $T^{2}$ denote the torus.

THeOREM 2. The classes $C_{p}$, for $0<p<1$, do not possess the GFP. In fact, if $N=M_{z+w}$ acting on $L^{2}\left(T^{2}\right)$, then for each $0<p<1$, there exists $X \in C_{p}$ such that $N X-X N \in C_{p}$ and yet $N^{*} X-X N^{*} \notin C_{p}$.

Proof. It is well known that $\left\{z^{i} w^{j}\right\}_{i, j=-\infty}^{\infty}$ is an orthonormal basis for $L^{2}\left(T^{2}\right)$. In this basis, the matrix of $M_{z+w}$ is $N=U \otimes I+I \otimes U$, where $U$ denotes the bilateral shift. Therefore the matrix of $M_{z+w}$ has nonzero blocks on the diagonal and lower diagonal, with all other blocks 0 . The diagonal blocks are bilateral shifts and the lower diagonal blocks are the identity $I$.

We choose $X$ to look exactly the same as $M_{z+w}$ except that the diagonal blocks will be weighted shifts and the lower diagonal blocks will be diagonal matrices. Let $y_{i j}$ denote the entries of the $j$ th diagonal block weighted shift, where $y_{i j}$ is the $i$ th weight on its lower diagonal (i.e. in the $(i, i-1)$ position). In the $j$ th lower diagonal block (in the $j$ th block row) let $x_{i j}$ denote the $i$ th diagonal entry (i.e. in the ( $i, i)$ position).

By computing the matrix entries of the commutators $N X-X N$ and $N^{*} X-$ $X N^{*}$ we obtain

$$
\begin{aligned}
\|N X-X N\|_{p}^{p} \leqslant & \sum_{i, j}\left|x_{i j}-x_{i j-1}\right|^{p}+\left|y_{i j}-y_{i-1 j}\right|^{p} \\
& +\left|\left(x_{i j}-x_{i-1 j}\right)-\left(y_{i j}-y_{i j-1}\right)\right|^{p}
\end{aligned}
$$

and

$$
\left\|N^{*} X-X N^{*}\right\|_{p}^{p} \geqslant \sum_{i, j}\left|x_{i j}-x_{i-1 j}\right|^{p}
$$

To accomplish that $\|N X-X N\|_{p}<\infty$ and $\left\|N^{*} X-X N^{*}\right\|_{p}=\infty$, it clearly suffices to obtain $x_{i j}, y_{i j}$ uniformly bounded, doubly indexed sequences (which insures that $X \in L(H \otimes H))$ such that

(1) $\sum_{i, j}\left|x_{i j}-x_{i j-1}\right|^{p}<\infty$,

(2) $\Sigma_{i, j}\left|y_{i j}-y_{i-1 j}\right|^{p}<\infty$,

(3) $\sum_{i, j}\left|\left(x_{i j}-x_{i-1 j}\right)-\left(y_{i j}-y_{i j-1}\right)\right|^{p}<\infty$,

(4) $\Sigma_{i, j}\left|x_{i j}-x_{i-1 j}\right|^{p}=\infty$.

We shall construct matrix arrays for $\left(x_{i j}\right)$ and $\left(y_{i j}\right)$ by taking the direct sums of finite arrays. Let $a_{n} A_{n}$ and $b_{n} B_{n}$ denote the square finite matrix arrays to be constructed. The scalars $a_{n}, b_{n}$ represent positive numbers we shall choose in order to guarantee that the entires of $\Sigma \oplus a_{n} A_{n}$ and $\Sigma \oplus b_{n} B_{n}$ remain uniformly bounded (to insure that $X$ be a bounded operator) and also to guarantee that they 
satisfy conditions (1)-(4). Choose $A_{n}$ and $B_{n}$ to be the matrix arrays

\begin{tabular}{|c|c|c|c|c|c|c|c|c|c|}
\hline \multicolumn{5}{|c|}{$A_{n}$} & & & \multicolumn{3}{|c|}{$B_{n}$} \\
\hline 00 & 0 & 0 & $\cdots 0$ & 00 & 00 & 0 & 0 & $\cdots$ & 00 \\
\hline $\begin{array}{ll}0 & 1\end{array}$ & 1 & 1 & $\cdots 1$ & 10 & $\begin{array}{ll}0 & 1\end{array}$ & 2 & . & $\cdots$ & $n$ \\
\hline 02 & 22 & 2 & $\cdots 2$ & 20 & $\begin{array}{ll}0 & 1\end{array}$ & 2 & $\cdots$ & $n$ & 0 \\
\hline 03 & 33 & 3 & $\cdots$ & 30 & 01 & 2 & $\cdots$ & $n$ & 0 \\
\hline • & $\cdot$ & $\cdot$ & $\cdots \cdot$ & . & $\cdot$ & - & $\cdot$ & $\cdots$ & • \\
\hline . $\cdot$ & $\cdot$ & • & $\cdots \quad$ & . $\cdot$ & . & . & . & $\cdots$ & . \\
\hline . & $\cdot$ & • & $\cdots$ & . $\cdot$ & . & . & . & $\cdots$ & . \\
\hline $0 n$ & $n$ & $n$ & $\cdots n$ & $n 0$ & $\begin{array}{ll}0 & 1\end{array}$ & 2 & . & $\cdots$ & $n$ \\
\hline 00 & 0 & 0 & $\cdots$ & 00 & 00 & 0 & . & & 0 \\
\hline
\end{tabular}

For convenience, let (1)-(4) also denote the corresponding sums in conditions (1)-(4). Let us investigate how (1)-(4) act on $A_{n}, B_{n}$ where $\left(x_{i j}\right)$ is $A_{n}$ and $\left(y_{i j}\right)$ is $B_{n}$ (considering them as two-way infinite arrays by extending them to have all other entries 0). By computing quantities (1)-(4) we see that

$$
\begin{aligned}
& \text { (1) }=(2)=2 \sum_{k=1}^{n} k^{p}<2 n \cdot n^{p}=2 n^{p+1}, \\
& \text { (3) }=2 \cdot n \cdot n^{p}=2 n^{p+1} \\
& \text { (4) }=\sum_{n^{2} \text { times }} 1+n \cdot n^{p}>n^{2} .
\end{aligned}
$$

In other words, (1), (2) and (3) applied to $A_{n}, B_{n}$ have orders of magnitude of $n^{p+1}$, whereas (3) grows with an order of magnitude of $n^{2}$. The clincher is that $p+1<2$ (since $p<1$ ). Set $a_{n}=1 / n^{3 / 2}=b_{n}$. Clearly $a_{n}, b_{n}<1 / n$ and so the entries of $a_{n} A_{n}$ and $b_{n} B_{n}$ are all less than or equal to 1 . Furthermore, applying (1)-(4) to $a_{n} A_{n}$ and $b_{n} B_{n}$ we obtain

$$
\begin{aligned}
& \text { (1) }<\left(1 / n^{3 / 2}\right)^{2} \cdot 2 n^{p+1}=2 / n^{2-p} \text { and similarly, } \\
& \text { (2) } \leqslant 2 / n^{2-p} \\
& \text { (3) }<2 / n^{2-p} \\
& \text { (4) } \geqslant n^{2} / n^{3} \geqslant 1 / n
\end{aligned}
$$

Now take $\left(x_{i j}\right)$ to be the two-way infinite matrix array $\Sigma \bigoplus a_{n} A_{n}$ and $\left(y_{i j}\right)$ to be $\Sigma \oplus b_{n} B_{n}$. However here we must just consider $a_{n} A_{n}$ and $b_{n} B_{n}$ as finite blocks surrounded by enough zeros so that when "pasted" together in the matrix they do not overlap and so (1)-(4) remain as before. The quantities (1)-(4) applied to $\Sigma \oplus a_{n} A_{n}$ and $\Sigma \oplus b_{n} B_{n}$ are simply the sum of the corresponding quantities (1)-(4) respectively, applied to $a_{n} A_{n}, b_{n} B_{n}$. This is because the sums (1)-(4) act independently on each finite block array in $\Sigma \bigoplus a_{n} A_{n}$ and $\Sigma \bigoplus b_{n} B_{n}$. Hence since 
$2-p>1$, we have

$$
\begin{gathered}
\text { (1) } \leqslant \sum \frac{2}{n^{2-p}}<\infty, \quad \text { (2) } \leqslant \sum \frac{2}{n^{2-p}}<\infty \\
\text { (3) } \leqslant \sum \frac{2}{n^{2-p}}<\infty, \quad \text { (4) } \geqslant \Sigma \frac{1}{n}=\infty . \quad \text { Q.E.D. }
\end{gathered}
$$

REMARK. These investigations have led to another kind of possible generalization of Fuglede's Theorem. Namely, let $\left\langle M_{k}\right\rangle$ and $\left\langle N_{k}\right\rangle$ be commuting sequences of normal operators (i.e., $M_{k} M_{j}=M_{j} M_{k}$ and $N_{k} N_{j}=N_{j} N_{k}$ ). Let $X \in L(H)$. The question is: Does $\sum_{k=1}^{N} M_{k} X N_{k}=0$ imply $\sum_{k=1}^{N} M_{k}^{*} X N_{k}^{*}=0$ ? Also if $\Sigma\left\|M_{k}\right\|\left\|N_{k}\right\|<\infty$, does $\sum_{1}^{\infty} M_{k} X N_{k}=0$ imply $\sum_{1}^{\infty} M_{k}^{*} X N_{k}^{*}=0$ ? Also are the corresponding statements true modulo the Hilbert-Schmidt class?

Thus far we know easily that all answers are yes when all normal operators are diagonalizable, and $\mathrm{C}$. Apostol settled the questions in the affirmative in the case $N=2$ using [6].

\section{REFERENCES}

1. B. A. Fuglede, A commutativity theorem for normal operators, Proc. Nat. Acad. Sci. U.S.A. 36 (1950), 35-40.

2. C. R. Putnam, Commutation properties of Hilbert space operators and related topics, Ergebnisse der Math. und ihrer Grenzgebiete, Band 36, Springer-Verlag, New York, 1967.

3. D. Voiculescu, Some results on norm ideal perturbations of Hilbert space operators, J. Operator Theory 2 (1979), 3-37.

4. G. Weiss, Commutators and operator ideals, Chapter 2, Dissertation, Univ. of Michigan, 1975.

5. The Fuglede Commutativity Theorem modulo the Hilbert-Schmidt class and generating functions for matrix operators. I, Trans. Amer. Math. Soc. 246 (1978), 193-209.

6. __ The Fuglede Commutativity Theorem modulo the Hilbert-Schmidt class and generating functions for matrix operators. II, J. Operator Theory (to appear).

7. S. K. Berberian, Note on a theorem of Fuglede and Putnam, Proc. Amer. Math. Soc. 10 (1959), 175-182.

8. B. Helton and R. Howe, Traces of commutators of integral operators, Acta Math. 135 (1975), 279.

Department of Mathematics, University of Cincinnati, Cincinnati, Oho 45221 